\title{
Cable test raises fears at fusion project
}

\section{Degradation of superconducting cables for the heart of the ITER fusion machine threatens to cause further delays.}

\section{BY GEOFF BRUMFIEL}

$\mathrm{S}$ cientists on three continents are scrambling to understand a potentially serious problem with superconducting cables destined for ITER, the world's largest fusion experiment. Nature has learned that preliminary tests of cable for ITER's powerful central magnet show that it degrades too quickly to be used. If unresolved, the problem threatens to further delay a project that has already suffered many years of technical and budgetary setbacks (see Nature 461, 855; 2009).

ITER is a $€ 15$-billion (US\$21-billion) collaboration designed to prove the viability of nuclear fusion as a power source. Now under construction in St-Paullez-Durance, France, and scheduled to start power-producing tests in 2026, the reactor will consist of a giant, doughnut-shaped vessel filled with hydrogen isotopes. Powerful magnetic fields generated by huge super-

\section{ITER'S BEATING HEART}

The superconducting cables of the central solenoid are a crucial part of the fusion reactor.

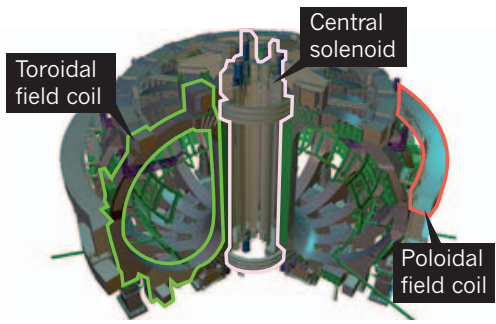

Mass production of the cable has been put on hold until a solution can be found, says Ryuji Yoshino, the head of Japan's Domestic Agency for ITER. He says that Japanese officials have not seen this kind of degradation in other projects, including earlier ITER test cables produced in 2000. He believes that the problem developed during the Swiss tests, which mimicked ITER's operating conditions by placing the cable in a strong but non-uniform magnetic field.

"I'm concerned, but we have a plan in place to solve the problem," says Ned Sauthoff, the head of the United States' contribution to ITER. Scientists at the Japan Proton Accelerator Research Complex in Ibaraki are now conducting neutron-scattering studies to measure the strain on the cable and to check for possible damage. Further experiments will be done in Switzerland to see whether the testing methodology is to blame. Meanwhile, neutron studies of the conducting coils surrounding the vessel will trap the isotopes as they are heated to the point of fusion.

The largest of these magnets is the central solenoid, a 13.5-metre-high, 4.1-metre-wide stack of six identical coils capable of generating a staggering 13 Tesla magnetic field (see 'ITER's beating heart'). By slowly pulsing the current in the superconducting cable, the solenoid generates a second current inside the hydrogen gas itself, which helps to confine the gas as it heats to fusion temperatures of more than 150 million ${ }^{\circ} \mathrm{C}$.

The solenoid will be built in the United States using superconducting niobium tin $\left(\mathrm{Nb}_{3} \mathrm{Sn}\right)$ cable manufactured in Japan. The cable is supposed to withstand 60,000 current pulses without trouble. But tests in November 2010 at the SULTAN facility, part of the Paul Scherrer Institute in Villigen, Switzerland, revealed serious degradation in a sample of cable after only 6,000 pulses. If confirmed, that would mean ITER could operate for only a fraction of its 20 -year lifetime with the existing cable. failed cable are also under way at the Spallation Neutron Source at Oak Ridge National Laboratory in Tennessee, along with X-ray tomography experiments at Lawrence Livermore National Laboratory in California, according to Sauthoff. In addition, other types of $\mathrm{Nb}_{3} \mathrm{Sn}$ cables are being tested to see if they are more resilient. "We're going to test until we get a success," he says.

Sauthoff hopes that the situation can be resolved by June. If a fix for the cable takes longer, then additional facilities could be tasked with manufacturing coils for the central solenoid to speed up production, but this would add extra expense to the project.

\section{CORRECTION}

The News story 'Traditional drug-discovery model ripe for reform' (Nature 471, 17-18; 2011) should have put the annual cost of the drug-development model discussed as US $\$ 200$ million, not $\$ 325$ million. 